\title{
Service evaluation of a digital behavioural change programme
}

\author{
Authors: Chris Edson, ${ }^{A}$ James Hampton ${ }^{B}$ and Edward Allen ${ }^{C}$
}

\section{Aims}

The purpose of this service evaluation was to analyse the efficacy of OurPath, a UK-based digital behavioural change programme, based on real-world data gathered in a commercial setting.

\section{Methods}

A quasi-experimental research design was used, which included longitudinal and pre-post tests of weight, activity levels and programme engagement outcomes.

In total, 98 potential participants with a mean body mass index of $31 \mathrm{~kg} / \mathrm{m}^{2}$ (obese) paid fees to enrol on OurPath, a digital behavioural change programme that combines a private online social network, daily structured educational content, health coaching, wireless scales and an activity tracker. Participants underwent a core 6-week intensive lifestyle change intervention and moved onto OurPath's Sustain programme, with follow-ups after 3 and 6 months.

Jamovi open-source statistics package was used to calculate statistical tests and $p$-values. One-way T-tests were used, with the null hypothesis being an average weight loss of $0 \%$ (ie no weight loss).

\section{Results}

A total of 77 participants met the inclusion criteria for the core programme and achieved weight loss from baseline of $5.3 \%$ in six weeks $(p<0.01)$. At the date of data analysis, 69 participants remained enrolled 3 months from their initial start date.

Of the 69 participants, 42 (61\%) had submitted a weight reading after 3 months and had achieved a mean weight loss of $6.7 \%$ $(p<0.01)$. Data available for 15 participants after 6 months $(52 \%$ of participants who had passed the 6 -month mark at the date of analysis) demonstrated a mean weight loss of $8.2 \%(p<0.01)$. Weight loss data were not available for all participants at the 3 and 6-month mark.

Authors: ${ }^{\mathrm{A} O u r P a t h}$, co-founder and CEO, London; ${ }^{\mathrm{B}}$ Bath and North East Somerset Clinical Commissioning Group, Bath; ${ }^{C}$ Birmingham Cross City Clinical Commissioning Group, Birmingham, UK

\section{Conclusion}

The OurPath online behavioural change programme achieved clinically significant weight loss results in a real-world setting. Online delivery platforms like OurPath could therefore offer an effective and scalable solution to tackle the UK's obesity and diabetes epidemics.

\section{Conflict of interest statement}

Chris Edson is a director of OurPath Ltd. James Hampton is a minority shareholder of OurPath Ltd. 